\title{
Evolving Fuzzy Classification System by a Quantum Particle Swarm Optimization Algorithm
}

\author{
Yunhui Zhu', a, Jun Sun ${ }^{1, b}$ \\ ${ }^{1}$ School of IOT, Jiangnan University, Wuxi 214122, China.

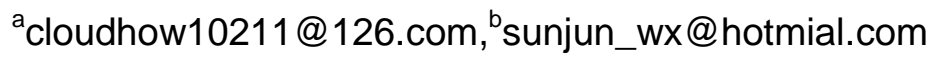

Keywords: fuzzy classification system, fuzzy rules, quantum evolving algorithm, quantum particle swarm optimization.

\begin{abstract}
This paper discusses how to construct a fuzzy classification system (FCS) effectively and accurately. In the FCS, the initial fuzzy rules are optimized with a quantum bit which has many unique advantages such as small population size, fast convergence, short training time and strong global search ability. After then, in order to accomplish the optimization for the fuzzy space partition and the number of fuzzy rules, this paper propose a method-quantum particle swarm optimization (QPSO) -to improve the initial FCS. The experiment result demonstrates that this method is more efficient than other methods without QPSO.
\end{abstract}

\section{Introduction}

Fuzzy rules-based classification for a practical problem employs the kinds of methods of extracting fuzzy rules ${ }^{[1,2]}$. The first one is to generate fuzzy inference rules based on the professional knowledge of experts in a particular field, while the second one is to reason out corresponding fuzzy IF-THEN rules from a set of given training data ${ }^{[3]}$. In this paper and other related literature, the so-called fuzzy classification employs the second method to generate a set of fuzzy classification rules. In this kind of methods, a training algorithm is needed to perform the training task or evolve fuzzy classification rules in other words. Many heuristic methods, such as genetic algorithm (GA), simulated annealing, quantum evolutionary algorithm (QEA) and quantum particle swarm optimization (QPSO), has been used to evolve the fuzzy rules of classifiers ${ }^{[4]}$ These methods act as the training algorithms to evolve or optimize the fuzzy rules to maximize the classification accuracy on the given training samples.

\section{Construction of fuzzy rules-based classification system}

The antecedent of the fuzzy IF-THEN rules should be determined while building fuzzy classification system model ${ }^{[5,6]}$. Then the determined antecedent trains the known data to achieve the consequent of the fuzzy rules. The antecedent of the fuzzy rules contains the number of input variables, the division of input space and the number of input space partitioning.

This paper establishes the initial classifier, and completes the modeling process on the basis of quantum bit ${ }^{[7]}$. The selection of number of the fuzzy rules depends on the individual of the population through quantum evolution. This paper adopts Gaussian membership functions and uses mesh division for dividing the input space.

Different classification methods of input space have corresponding antecedent of the fuzzy IF-THEN rules, so as to generate different fuzzy classification models ${ }^{[13]}$. So in order to transform training samples into correct classifier and keep the number of fuzzy rules to a minimum, the common method is to use individual to represent antecedent of the fuzzy rules, and then the method can get the consequent and corresponding categories through training data by evolutionary algorithm. The evaluation index of a fuzzy classification system includes the classification accuracy of training data, the number of input variables and the number of fuzzy rules. 


\section{Optimization for fuzzy classification system}

\subsection{Theory of qubit expression}

In the normal or classical computing concept, information is represented in bits where each bit must hold a value of either 0 or 1 . However, in quantum computing, information is instead represented by a qubit in which a value of a single qubit could be 0,1 , or a superposition of both ${ }^{[8]}$. Superposition allows the possible states to represent both 0 and 1 simultaneously based on its probability. The quantum state is modeled by the Hilbert space of wave functions and is defined as:

$|\psi\rangle=\alpha|1\rangle+\beta|0\rangle(3.5)$

where $\alpha$ and $\beta$ are complex numbers defining probabilities at which the corresponding state is likely to appear when a qubit collapses, for instance, when reading or measuring. Probability fundamentals stated that $|\alpha|^{2}+|\beta|^{2}=1$, where $|\alpha|^{2}$ gives the probability that a qubit is in the OFF (0) state and $|\beta|^{2}$ gives the probability that a qubit is in the ON (1) state. The probability $\left[\begin{array}{l}\alpha \\ \beta\end{array}\right]$ of can be represented as quantum angle $\theta$, where satisfies the probability fundamental of $|\sin (\theta)|^{2}+|\cos (\theta)|^{2}=1$.

\subsection{Quantum particle swarm optimization algorithm}

In the QPSO with M individuals, each individual is treated as a volume-less and spin-less particle in the $\mathrm{N}$-dimensional space, with the current position vector of particle $\mathrm{i}$ at the nth iteration represented by $X_{i, n}=\left(X_{i, n}^{1}, X_{i, n}^{2}, \mathrm{~L}, X_{i, n}^{N}\right)$. Besides, each particle i also has a vector $P_{i, n}=\left(P_{i, n}^{1}, P_{i, n}^{2}, \mathrm{~L}, P_{i, n}^{N}\right)$, which is the best previous position (the position giving the best objective function value or fitness value) of particle $\mathrm{i}$ called personal best(pbest) position. The whole swarm has a vector $G_{n}=\left(G_{n}^{1}, G_{n}^{2}, \mathrm{~L}, G_{n}^{N}\right)$, which is the position of the best particle among all the particles in the population and called global best (gbest) position ${ }^{[9]}$. If this paper consider the following minimization problem:

$$
\text { Minimize } f(X) \text {, s.t. } X \in S \subseteq R^{N} \text {, }
$$

Where $f(X)$ is an objective function continuous almost everywhere and $S$ is the feasible space, then $P_{i, n}$ can be updated by

$$
P_{i, n}=\left\{\begin{array}{ccc}
X_{i, n} & \text { if } & f\left(X_{i, n}\right)<f\left(P_{i, n-1}\right) \\
P_{i, n-1} & \text { if } & f\left(X_{i, n}\right) \geq f\left(P_{i, n-1}\right)
\end{array},\right.
$$

and accordingly $G_{n}$ can be found by $G_{n}=P_{g, n}$, where $g=\arg \min _{1 \leq i \leq M}\left[f\left(P_{i, n}\right)\right]$.

The particle in the QPSO updates its position the following equation:

$$
X_{i, n+1}^{j}=p_{i, n}^{j} \pm \alpha\left|X_{i, n}^{j}-C_{n}^{j}\right| \ln \left(1 / u_{i, n+1}^{j}\right),
$$

for $i=1,2, \mathrm{~L} M ; j=1,2 \mathrm{~L}, N$, where

$$
p_{i, n}^{j}=\varphi_{i, n}^{j} P_{i, n}^{j}+\left(1-\varphi_{i, n}^{j}\right) G_{n}^{j}, \varphi_{i, n}^{j} \sim U(0,1) .
$$

In (1), $C_{n}=\left(C_{n}^{1}, C_{n}^{2}, \mathrm{~L}, C_{n}^{N}\right)$ is called mean best (mbest) position defined by the average of the pbest positions of all particles, i.e., $C_{n}^{j}=(1 / M) \sum_{i=1}^{M} P_{i, n}^{j} \quad(1 \leq j \leq N)$. $u_{i, n+1}^{j}$ is a sequence of random numbers uniformly distributed on $(0,1)$, and parameter $\alpha$ is known as the contraction-expansion (CE) coefficient, which can be adjusted to balance the local and global search of the algorithm during the optimization process. 


\subsection{The principle for qubit calculation with particle swarm optimization}

The quantum computation also has been extended to PSO and this is known as Quantum-inspired Particle Swarm Optimization (QPSO). The main idea of QPSO is to update the particle position represented as a quantum angle $\theta$. The common velocity update equation in conventional PSO is modified to get a new quantum angle which is translated to the new probability of the qubit by using the following formula:

$$
\Delta \theta_{n}=w^{*} \Delta \theta_{n-1}+c_{1} * \operatorname{rand}() *\left(\theta_{\text {gbest }_{n}}-\theta_{n}\right)+c_{2} * \operatorname{rand}() *\left(\theta_{\text {pbest }_{n}}-\theta_{n}\right)
$$

Based on the new $\theta$ velocity, the new probability of $\alpha$ and $\beta$ is calculated using a rotation gate as follows:

$$
\left[\begin{array}{l}
\alpha \\
\beta
\end{array}\right]=\left[\begin{array}{cc}
\cos (\Delta \theta) & -\sin (\Delta \theta) \\
\sin (\Delta \theta) & \cos (\Delta \theta)
\end{array}\right]\left[\begin{array}{c}
\alpha_{t-1} \\
\beta_{t-1}
\end{array}\right]
$$

In a feature selection task, each qubit denoted as quantum angle $\theta$, represents one feature. In this case, the collapse qubit value 1 represents features selected while value 0 represents those not selected.

\subsection{0ptimization for fuzzy classification system Based on qubit expression and QPSO}

Initialization is conducted for building the first generation of populations in the fuzzy system model. Determination of the input space partition is based on using random individual way. The category and confidence of fuzzy rules depend on the calculation training data in the input space. For the following calculation of each generation, QEA makes division according to each input space, and then determines the class of fuzzy rules and confidence according to the section 2.

In the fuzzy classification system, this paper encode a certain size of population in the form of quantum on the basis of qubit expression and QPSO, and the population size is determined according to the input data. Then select the appropriate fitness function according to the study data, and calculate the fitness function value of each individual population.

In the each iteration, the population must be updated. The core of the population updating is the quantum rotation and quantum crossover, which generates a new population of individuals and makes the original model evolve to the direction of the optimal solution after quantum operations gradually.

(1) Encoding method

Canonical fuzzy classification system directly builds fuzzy rules based on expertise or experience, while ensuring certain rationality. But it is difficult to achieve the purpose of rapid establishment of classification systems. And also for more complex subjects, constructed classification system will appear blurred rules larger redundancy situation. Population coding and qubit choosing could avoid these problems.

Each individual in the population for QEA contains for all antecedent of the fuzzy IF-THEN rules in a fuzzy classification system. After binary coding, each individual include two components, named features control and fuzzy division control, respectively. The two part together act in each individual input data to divide the input space.

(2) The selection of fitness function

Fuzzy rules number is an important indicator of the fuzzy logic model and also is an important aspect of explanatory. Therefore, the model explanatory must be fully considered while establishing fuzzy classification system model, and with the same time while it is joined into choose membership function and fitness function.

Fuzzy classification system based on qubit expression and QPSO emphasis on finding the optimal individual, increasing the classification accuracy of the classification system as far as possible, namely classified samples correctly to the maximum. At the same time this paper should keep fuzzy classification model contain fewer fuzzy rules number. Fitness functions as shown below:

$$
F_{1}=W_{1} \cdot f_{1}-W_{2} \cdot N_{r}
$$

$f_{1}$ is the number of training samples classified correctly. $N_{r}$ is the number of fuzzy rules in the 
fuzzy classification system. $W_{1}$ and $W_{2}$ presents the weight of $f_{1}$ and $N_{r}$, respectively.

In fuzzy classification system model, the requirement of the classification ability of fuzzy system is higher than the rules number of the system. So this paper sets $W_{1}$ and $W_{2}$ into $0<W_{2}<<W_{1}$.

(3) Population Updating

In the theory of qubit expression, the use of quantum gates transforming the matrix to achieve the transfer between the various states, and it can make use of quantum revolving door rotation angle to complete the process of chromosome variation in process variation to produce new individuals, so as to achieve the purpose of speeding up the convergence .Update the expression of the probability $\left[\begin{array}{l}\alpha \\ \beta\end{array}\right]$ with the following formula:

$$
\left[\begin{array}{l}
\alpha \\
\beta
\end{array}\right]=\left[\begin{array}{cc}
\cos (\Delta \theta) & -\sin (\Delta \theta) \\
\sin (\Delta \theta) & \cos (\Delta \theta)
\end{array}\right]\left[\begin{array}{c}
\alpha_{t-1} \\
\beta_{t-1}
\end{array}\right]
$$

\section{Experimental Results and Discussion}

\subsection{The data set of Iris}

The first simulation example is based on the Iris data set. The Iris is a typical classification problem; is generally used for assessment of the classification algorithm. So the experiment is based on Iris data for simulation.

Iris data from consists of 150 four dimensional samples (calyx length, calyx width, petal length and petal width).The data is divided into three categories. 50 samples constitute a category. The data from the first and the second category could be separated completely, but there is a cross between the second and the third category.

Modeling three kinds of four dimensional data: take value length and value width as two input variables namely $\mathrm{x}_{3}$ and $\mathrm{x}_{4}, \mathrm{x}_{3}$ use four Gaussian membership to divide the input variable space, $\mathrm{x}_{4}$ use four a Gaussian membership to divide the input variables, set up 16 fuzzy rules totally. According to QEA, the choice of fuzzy rule to Initial fuzzy classification system model: population evolution algebra is set to 100 generation, individual number is 20 , the value of fitness function is $W_{N C P}=100, W_{S}=1$, overlap factor $\mu=0.2$.
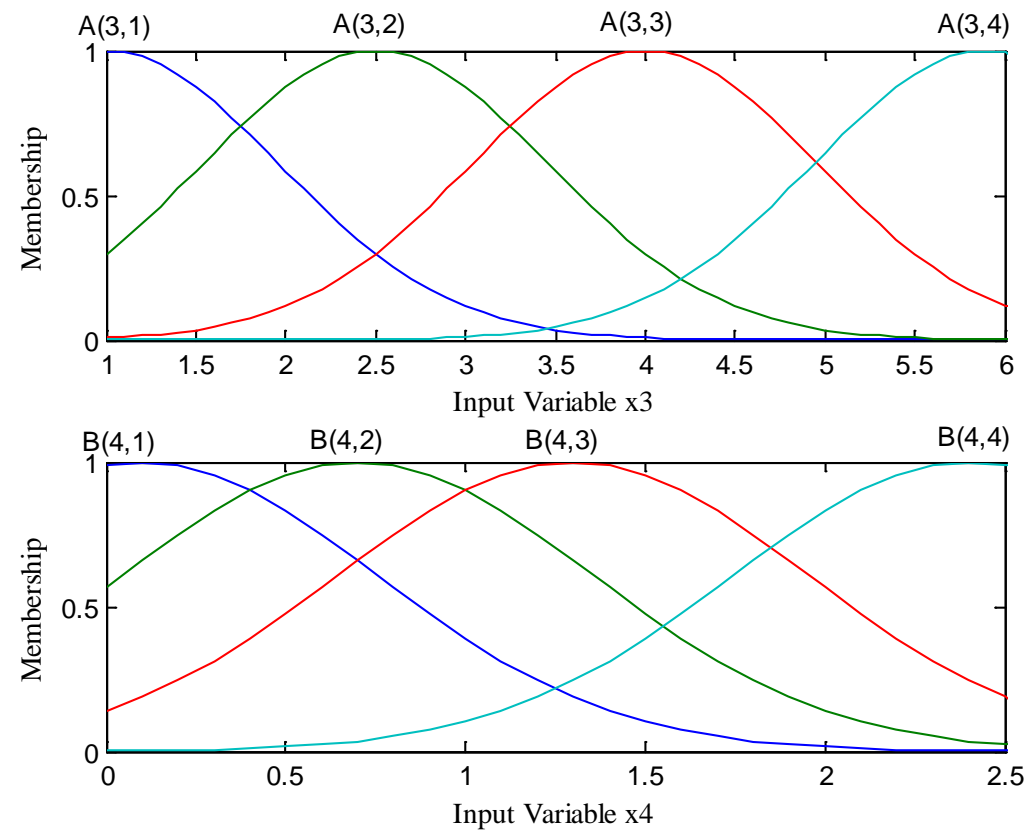

Fig.4-1 membership function of Iris initial fuzzy classification system

Iris data set contains two invalid fuzzy rules during establishing the initial fuzzy classification 
system model, and suggests that there is no data under the two fuzzy rules. So the initial fuzzy classification model is:

$\mathrm{R} 1: \mathrm{IFx}_{3}$ is $\mathrm{A}(3,1)$ and $_{4}$ is $\mathrm{B}(4,1)$,THEN $\mathrm{x}$ belongs to Class 1 with $\mathrm{C}=0.9974$

$\mathrm{R} 2: \mathrm{IFx}_{3}$ is $\mathrm{A}(3,1)$ and $_{4}$ is $\mathrm{B}(4,2)$,THEN $\mathrm{x}$ belongs to Class 1 with $\mathrm{C}=0.9279$

R3:IFx $x_{3}$ is $A(3,1) a n d x_{4}$ is $B(4,3)$, THEN $x$ belongs to Class 1 with $C=0.9385$

$\mathrm{R} 16: \mathrm{IFx}_{3}$ is $\mathrm{A}(3,4)$ and $x_{4}$ is $\mathrm{B}(4,3), \mathrm{THEN} \mathrm{x}$ belongs to Class 3 with $=0.8393$

The simulation experiment of the Iris data set shows that the initial fuzzy classification system based on the QEA. The initial fuzzy classification system adopted two characteristic variables: input variable $\mathrm{x}_{3}$ and input variablesx $\mathrm{x}_{4}$ (petal length and petal width), consists of 16 fuzzy rules. The rule with category number displaying "NULL" represents the uncertainty associated with rules $\mathrm{R}_{\mathrm{ij}}$. On the other hand, it presents that the rules have no relevant data or there is a small amount of data. The rule with category number displaying "NULL" represents its corresponding confidence values "zero", and is called namely dummy-rules. Therefore the correct classification samples number of the initial fuzzy classification model based on QEA can reach 143, due to the second and the third category of data exist intersections.

\subsection{The data set of Wine}

The second simulation example is conducted with wine data sets. The Wine consists of chemical composition of three different wines, 13 attributes of178 groups, namely the $178 * 13$ dimension data.QEA is used to construct the fuzzy classification system model.13 attributes are: alcohol $\left(x_{1}\right)$, malic acid $\left(x_{2}\right)$, ash content $\left(x_{3}\right)$, ash contentalkalinity $\left(x_{4}\right)$, magnesium $\left(x_{5}\right)$, total phenol $\left(x_{6}\right)$, falconoid $\left(x_{7}\right)$, phenolic $\left(x_{8}\right)$, anthocyanin $\left(x_{9}\right)$, color intensity $\left(x_{10}\right)$, chroma $\left(x_{11}\right)$, OD280/OD315 dilute wine $\left(x_{12}\right)$ and amino acid $\left(x_{13}\right)$. Among of them, alcohol $\left(x_{1}\right)$, flavonoid $\left(x_{7}\right)$, $\operatorname{chroma}\left(x_{11}\right)$, OD280/OD315 dilute wine $\left(x_{12}\right)$ and amino acid $\left(x_{13}\right)$ are input variables. This paper divides the input space of five input variables using the Gaussian membership function. The division result showed in the following figure 4-2.
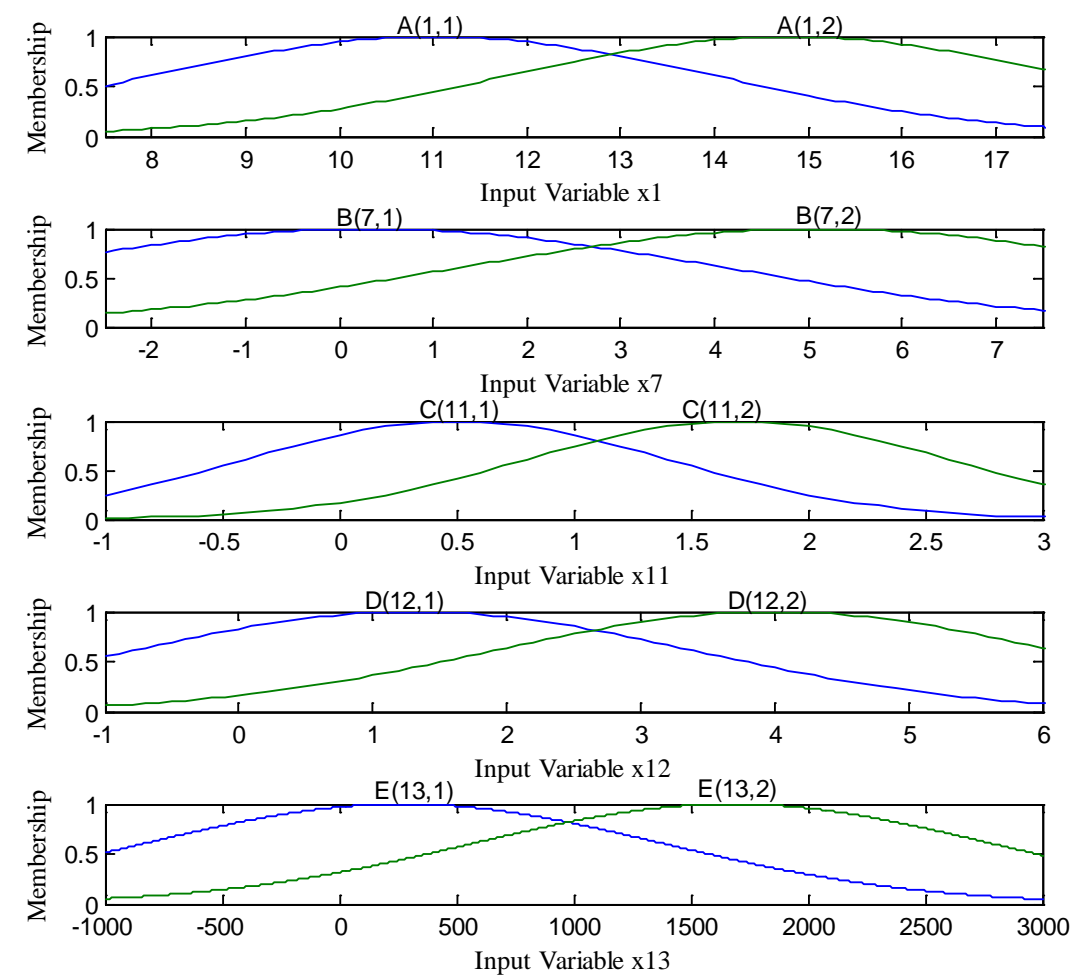

Fig. 4-2 membership function of wine initial fuzzy classification system 
The initial fuzzy classification system model concluded with the wine data presents as follows:

$\mathrm{R} 1: \mathrm{IFx}_{1}$ is $\mathrm{A}(1,1)$ and $\mathrm{x}_{7}$ is $\mathrm{B}(7,1)$ and $\mathrm{x}_{11}$ is $\mathrm{C}(11,1)$

and $x_{12}$ is $D(12,1)$ and $x_{13}$ is $A(13,1)$

THEN $x$ belongs to Class 2 with $C F=0.1487$

$\mathrm{R} 2: \mathrm{IFx}_{1}$ is $\mathrm{A}(1,1)$ and $_{7}$ is $\mathrm{B}(7,1)$ and $_{11}$ is $\mathrm{C}(11,1)$ and $x_{12}$ is $\mathrm{D}(12,1)$ and $_{13}$ is $\mathrm{E}(13,1)$

THEN $x$ belongs to Class 3 with $\mathrm{CF}=0.0804$

$\mathrm{R} 3: \mathrm{IFx}_{1}$ is $\mathrm{A}(1,1)$ and $_{7}$ is $\mathrm{B}(7,1)$ and $_{11}$ is $\mathrm{C}(11,1)$ and $\mathrm{x}_{12}$ is $\mathrm{D}(12,2)$ and $\mathrm{x}_{13}$ is $\mathrm{E}(13,1)$

THEN $x$ belongs to Class 2 with $\mathrm{CF}=0.2678$

\section{R32: $\operatorname{IFx}_{1}$ is $A(1,2)$ and $_{7}$ is $B(7,2)$ and $_{11}$ is $C(11,2)$ and $x_{12}$ is $\mathrm{D}(12,2)$ and $\mathrm{x}_{13}$ is $\mathrm{E}(13,2)$ \\ THEN $x$ belongs to Class 1 with $\mathrm{CF}=0.4385$}

The initial fuzzy classification system adopts five characteristic variables and consists of 32 fuzzy rules. The rule with category number displaying "NULL" represents the uncertainty associated with rules $\mathrm{R}_{\mathrm{ij}}$. On the other hand, it presents the rules have no relevant data or there is a small amount of data. The rule with category number displaying "NULL" represents its corresponding confidence values "zero", and is called namely dummy-rules. Therefore, the correct classification samples number of the initial fuzzy classification model based on QEA can reach 161.

\subsection{The result of the experiment}

A. Experiment 1

The first simulation example: after the optimization of the Iris data, getting the binary code this paper obtained 9 fuzzy rules, including 1, 2, 5, 6, 10, 11, 12, 15and 16.The optimized fuzzy rules as follows:

Table 4-1 the number of Iris fuzzy rules after optimizing

$(\mathrm{RU}=$ rules, Cen $=$ center, $\mathrm{L}=$ left width, $\mathrm{R}=$ right width, $\mathrm{C}=$ classification, $\mathrm{CF}=$ confidence $)$

\begin{tabular}{|l|l|l|l|l|l|l|l|l|}
\hline \multirow{2}{*}{ RU } & \multicolumn{4}{|l|}{ Input $\mathrm{x}_{3}$} & \multicolumn{2}{l|}{ Input $\mathrm{x}_{4}$} & \multirow{2}{*}{ C } & \multirow{2}{*}{ CF } \\
\cline { 2 - 9 } & Cen & $\mathrm{L}$ & $\mathrm{R}$ & $\mathrm{Cen}$ & $\mathrm{L}$ & $\mathrm{R}$ & & \\
\hline 1 & 1 & 0 & 0.9654 & 0.1 & 0 & 0.6548 & 1 & 1.0 \\
\hline 2 & 1 & 0 & 0.9654 & 0.7 & 0.6548 & 0.6548 & 1 & 1.0 \\
\hline 5 & 2.5 & 0.9654 & 0.9654 & 0.1 & 0 & 0.6548 & 1 & 1.0 \\
\hline 6 & 2.5 & 0.9654 & 0.9654 & 0.7 & 0.6548 & 0.6548 & 1 & 1.0 \\
\hline 10 & 4.0 & 0.9654 & 0.9654 & 0.7 & 0.6548 & 0.6548 & 2 & 1.0 \\
\hline 11 & 4.0 & 0.9654 & 0.9654 & 1.3 & 0.6548 & 0.6548 & 2 & 0.8935 \\
\hline 12 & 4.0 & 0.9654 & 0.9654 & 2.4 & 0.6548 & 0 & 3 & 1.0 \\
\hline 15 & 5.9 & 0.9654 & 0 & 1.3 & 0.6548 & 0.6548 & 3 & 0.7510 \\
\hline 16 & 5.9 & 0.9654 & 0 & 2.4 & 0.6548 & 0 & 3 & 1.0 \\
\hline
\end{tabular}

Table 4-2 represents the data contrast between the initial classification system model and the optimized one of the fuzzy classification system model through the QPSO for the Iris data set, including the number of input variables, the number of the fuzzy set, the number of used fuzzy rules and the final number of samples which has been classified correctly.

Table 4-2 data comparison before and after Iris optimization

\begin{tabular}{|l|l|l|l|l|l|}
\hline $\begin{array}{l}\text { Fuzzy Classification } \\
\text { system(FCS) }\end{array}$ & $\begin{array}{l}\text { The Number } \\
\text { of Input } \\
\text { Variables }\end{array}$ & $\begin{array}{l}\text { The Number } \\
\text { of FS }\end{array}$ & $\begin{array}{l}\text { The } \\
\text { Number of } \\
\text { FR }\end{array}$ & $\begin{array}{l}\text { The number of } \\
\text { Correct Individuals }\end{array}$ & The Accuracy \\
\hline Initial FCS & 2 & 8 & 16 & 138 & $92.45 \%$ \\
\hline Post optimality FCS & 2 & 8 & 9 & 145 & $96.67 \%$ \\
\hline
\end{tabular}

B. Experiment2

The second simulation example: after the optimization of the Wine data in table 2-2,getting the binary code rule, this paper obtain 10 fuzzy rules, including1,2,3, 5,6, 7, 8, 11, 15, and16. The optimized fuzzy rules as follows: 
Table 4-3 the number of fuzzy rules after wine optimizes

$(\mathrm{RU}=$ rules, Cen $=$ center, $\mathrm{L}=$ left width, $\mathrm{R}=$ right width $\mathrm{C}=$ classification, $\mathrm{CF}=$ confidence $)$

\begin{tabular}{|c|c|c|c|c|c|c|c|c|c|c|c|c|c|c|c|c|c|}
\hline \multirow{2}{*}{ RU } & \multicolumn{3}{|c|}{ Input $\mathrm{x}_{1}$} & \multicolumn{3}{|c|}{ Input $x_{7}$} & \multicolumn{3}{|c|}{ Input $\mathrm{x}_{11}$} & \multicolumn{3}{|c|}{ Input $x_{12}$} & \multicolumn{3}{|c|}{ Input $x_{13}$} & \multirow{2}{*}{$C$} & \multirow{2}{*}{ CF } \\
\hline & Cen & $\mathrm{L}$ & $\mathrm{R}$ & Cen & $\mathrm{L}$ & $\mathrm{R}$ & Cen & $\mathrm{L}$ & $\mathrm{R}$ & Cen & $\mathrm{L}$ & $\mathrm{R}$ & Cen & $\mathrm{L}$ & $\mathrm{R}$ & & \\
\hline 1 & 11 & 0 & 3.0 & 0.3 & 0 & 3.8 & 0.5 & 0.9 & 0 & 1.3 & 0 & 2.1 & 278 & 0 & 1105 & 3 & 1.0 \\
\hline 2 & 11 & 0 & 3.0 & 0.3 & 0 & 3.8 & 0.5 & 0.9 & 0 & 1.3 & 0 & 2.1 & 1680 & 1105 & 0 & 3 & 1.0 \\
\hline 3 & 11 & 0 & 3.0 & 0.3 & 0 & 3.8 & 0.5 & 0 & 0.9 & 4 & 2.1 & 0 & 278 & 0 & 1105 & 2 & 0.9233 \\
\hline 5 & 11 & 0 & 3.0 & 0.3 & 0 & 3.8 & 1.7 & 0.9 & 0 & 1.3 & 0 & 2.1 & 278 & 0 & 1105 & 2 & 0.9457 \\
\hline 6 & 11 & 0 & 3.0 & 0.3 & 0 & 3.8 & 1.7 & 0.9 & 0 & 1.3 & 0 & 2.1 & 1680 & 1105 & 0 & 2 & 1.0 \\
\hline 7 & 11 & 0 & 3.0 & 0.3 & 0 & 3.8 & 1.7 & 0.9 & 0 & 4 & 2.1 & 0 & 278 & 0 & 1105 & 2 & 0.9430 \\
\hline 8 & 11 & 0 & 3.0 & 0.3 & 0 & 3.8 & 1.7 & 0.9 & 0 & 4 & 2.1 & 0 & 1680 & 1105 & 0 & 1 & 0.9882 \\
\hline 11 & 11 & 0 & 3.0 & 5.1 & 3.8 & 0 & 0.5 & 0 & 0.9 & 4 & 2.1 & 0 & 278 & 0 & 1105 & 2 & 0.9825 \\
\hline 15 & 11 & 0 & 3.0 & 5.1 & 3.8 & 0 & 1.7 & 0.9 & 0 & 4 & 2.1 & 0 & 278 & 0 & 1105 & 1 & 0.9900 \\
\hline 16 & 11 & 0 & 3.0 & 5.1 & 3.8 & 0 & 1.7 & 0.9 & 0 & 4 & 2.1 & 0 & 1680 & 1105 & 0 & 1 & 0.9977 \\
\hline
\end{tabular}

Table 4-4 represents the data contrast between the initial classification system model and the optimized ones of the fuzzy classification system model through the QPSO for the wine data set, including the number of input variables, the number of the fuzzy set, the number of used fuzzy rules and the final number of samples which has been classified correctly.

Table 4-4 data comparison before and after wine optimization

\begin{tabular}{|l|l|l|l|l|l|}
\hline $\begin{array}{l}\text { Fuzzy Classification } \\
\text { system(FCS) }\end{array}$ & $\begin{array}{l}\text { The Number } \\
\text { of Input } \\
\text { Variables }\end{array}$ & $\begin{array}{l}\text { The Number } \\
\text { of FS }\end{array}$ & $\begin{array}{l}\text { The Number of } \\
\text { FR }\end{array}$ & $\begin{array}{l}\text { The number of } \\
\text { Correct Individuals }\end{array}$ & $\begin{array}{l}\text { The } \\
\text { Accuracy }\end{array}$ \\
\hline Initial FCS & 5 & 10 & 32 & 156 & $87.87 \%$ \\
\hline Post optimality FCS & 5 & 10 & 10 & 163 & $91.57 \%$ \\
\hline
\end{tabular}

Table 4-5 focuses on comparison experiments to two data sets with three experimental methods: classification system model with the direct fuzzy system, fuzzy classification model using genetic algorithm and establish fuzzy classification system model using QPSO respectively. The following is three kinds of experimental comparison results:

Table 4-5Experimental comparison of three kinds of fuzzy classification method

\begin{tabular}{|c|c|c|c|c|c|c|}
\hline $\begin{array}{c}\text { Modeling } \\
\text { Method }\end{array}$ & $\begin{array}{c}\text { Data } \\
\text { Set }\end{array}$ & $\begin{array}{c}\text { Number } \\
\text { of fuzzy } \\
\text { rules }\end{array}$ & $\begin{array}{c}\text { The number } \\
\text { of the } \\
\text { optimized } \\
\text { rules }\end{array}$ & $\begin{array}{c}\text { The correct } \\
\text { classification } \\
\text { number }\end{array}$ & $\begin{array}{c}\text { The } \\
\text { sample } \\
\text { number }\end{array}$ & $\begin{array}{c}\text { The } \\
\text { classification } \\
\text { accuracy }\end{array}$ \\
\hline \multirow{2}{*}{$\begin{array}{c}\text { Direct } \\
\text { FCS }\end{array}$} & Iris & $4^{4}=256$ & 26 & 143 & 150 & $95.33 \%$ \\
\cline { 2 - 7 } & wine & $2^{13}=8192$ & 70 & 170 & 178 & $95.51 \%$ \\
\hline $\begin{array}{c}\text { FCS } \\
\text { Based on } \\
\text { GA }\end{array}$ & Iris & $4^{4}=256$ & 12 & 142 & 150 & $94.67 \%$ \\
\cline { 2 - 7 } & wine & $2^{5}=32$ & 12 & 160 & 178 & $89.89 \%$ \\
\hline $\begin{array}{c}\text { FCS } \\
\text { Based on } \\
\text { QPSO }\end{array}$ & Iris & $4^{2}=16$ & 9 & 146 & 150 & $97.33 \%$ \\
\cline { 2 - 7 } & wine & $2^{5}=32$ & 10 & 172 & 178 & $96.63 \%$ \\
\hline
\end{tabular}

Table 4-5 shows the fuzzy classification system model. The model data volume is large. The fuzzy rules number of the initial classification model will present increasing exponentially with the increasing of attributes number. The model has the advantage of high accuracy, but its explanatory 
is the bad. Using genetic algorithm to establish the fuzzy classification model is an optimization process conducted on the basis of the direct model modeling. It simplifies the structure of the initial classification model, so as to improve the model's explanation. The classification system model optimized by QPSO further simplifies its initial classification model structure on the basis of genetic algorithm. On the premise of guaranteeing the accuracy, the QPSO further simplifies model structure, so as to improve the explanatory of fuzzy classification model.

\section{Conclusion}

This paper first proposes fuzzy classification system model based on the theory of the qubit expression. The classification model can optimize the number of input variables and simplify the input variable, avoid the problem of "dimension disaster". After completing the building of initial fuzzy classification system, continue to use QPSO to optimize fuzzy rules. Then this paper verifies the feasibility of the method through four simulation experiments. Four data sets are Iris data sets, wine data sets, glass data sets and seeds data sets, respectively. The experimental results verify the feasibility of the proposed method, also has obtained the ideal effect. Finally, this paper use three experiments to compare the classification model data, regarding four data sets as samples, under the premise of the accuracy and explanatory for classification model, respectively.

\section{References}

[1]Zadeh L A. Fuzzy logic[J] . Computer, 1988, 21(4): 83-93.

[2]Zadeh L A. Fuzzy sets[J] . Information and control, 1965, 8(3): 338-353.

[3] Abe S, Lan M S. A method for fuzzy rules extraction directly from numerical data and its application to pattern classification[J] . Fuzzy Systems, IEEE Transactions on, 1995, 3(1): 18-28.

[4] Alexiev K M, Georgieva O I. Improved fuzzy clustering for identification of Takagi-Sugeno model[C] //Intelligent Systems, 2004. Proceedings. 2004 2nd International IEEE Conference. IEEE, 2004, 1: 213-218.

[5] Angelov P, Zhou X, Klawonn F. Evolving fuzzy rule-based classifiers[C] //Computational Intelligence in Image and Signal Processing, 2007. CIISP 2007. IEEE Symposium on. IEEE, 2007: 220-225.

[6] Castellano G, Fanelli A M. Modeling fuzzy classification systems with compact rule base[J] . Computational Intelligence for Modelling, Control \& Automation: Evolutionary Computation \& Fuzzy Logic for Intelligent Control, Knowledge Acquisition \& Information Retrieval, 1999, 2: 210.

[7] Cheng W Y, Juang C F. An incremental support vector machine-trained TS-type fuzzy system for online classification problems[J] . Fuzzy Sets and Systems, 2011, 163(1): 24-44.

[8] Dias J M, Dourado A. A self-organizing fuzzy controller with a fixed maximum number of rules and an adaptive similarity factor[J] . Fuzzy Sets and Systems, 1999, 103(1): 27-48.

[9] Han K H, Kim J H. Genetic quantum algorithm and its application to combinatorial optimization problem[C] //Evolutionary Computation, 2000. Proceedings of the 2000 Congress on. IEEE, 2000, 2: $1354-1360$.

[10] Han K H, Kim J H. Quantum-inspired evolutionary algorithm for a class of combinatorial optimization[J] . Evolutionary Computation, IEEE Transactions on, 2002, 6(6): 580-593.

[11] Sun,J., Feng,B., Xu,W.B., 2004. Particle Swarm Optimization with Particles Having Quantum Behavior. Proc. 2004 Congress on Evolutionary Computation. Piscataway, NJ. 325-331

[12] Sun,J., Xu,W.B., Feng,B., 2005. Adaptive Parameter Control for Quantum-behaved Particle Swarm Optimization on Individual Level. 2005 IEEE International Conference on Systems, Man and Cybernetics. Waikoloa, Hawaii, 3049-3054. 
[13] Kennedy,J., 2003. Bare bones particle swarms. In: Proceedings of the 2003 IEEE swarm intelligence symposium, 80-87.

[14] Kennedy,J., Eberhart,R., 1995. Particle Swarm Optimization. Proc. IEEE int. Conf. On Neural Network. 1942-1948.

[15] Hui C, Jiashu Z, Chao Z. Chaos updating rotated gates quantum-inspired genetic algorithm[C] //Communications, Circuits and Systems, 2004. ICCCAS 2004. 2004 International Conference on. IEEE, 2004, 2: 1108-1112.

[16] Ishibuchi $\mathrm{H}$, Yamamoto $\mathrm{T}$. Effects of three-objective genetic rule selection on the generalization ability of fuzzy rule-based systems[C] //Evolutionary Multi-Criterion Optimization. Springer Berlin Heidelberg, 2003: 608-622.

[17] Ishibuchi H, Yamamoto T. Fuzzy Rule Selection By Data Mining Criteria And Genetic Algorithms[C] //GECCO. 2002: 399-406.

[18] Juang C F, Hsiao C M, Hsu C H. Hierarchical cluster-based multispecies particle-swarm optimization for fuzzy-system optimization[J] . Fuzzy Systems, IEEE Transactions on, 2010, 18(1): 14-26.

[19] Kao,Y., Zahara,E., Kao,I.W., 2008. A Hybridized Approach to Data Clustering. Expert Systems with Applications, 34(3), 1754-1762.

[20] Krishna,K., Murty,M., 1999. Genetic K-means algorithm, IEEE Transactions on Systems, Man and Cybernetics-Part B: Cybernetics, 29, 433-439.

[21] Krohling,R.A., 2004. Gaussian swarm: a novel particle swarm optimization algorithm. In: Proceedings of the 2004 IEEE conference on cybernetics and intelligent systems, 272-276.

[22] Kromer P, Platos J, Snasel V, et al. Fuzzy classification by evolutionary algorithms[C] //Systems, Man, and Cybernetics (SMC), 2011 IEEE International Conference on. IEEE, 2011: 313-318.

[23] Lee H M, Chen C M, Chen J M, et al. An efficient fuzzy classifier with feature selection based on fuzzy entropy[J] . Systems, Man, and Cybernetics, Part B: Cybernetics, IEEE Transactions on, 2001, 31(3): 426-432.

[24] Zhang G, Jin W, Hu L. A novel parallel quantum genetic algorithm[C] //Parallel and Distributed Computing, Applications and Technologies, 2003. PDCAT'2003. Proceedings of the Fourth International Conference on. IEEE, 2003: 693-697.

[25] Lemos A, Caminhas W, Gomide F. Adaptive fault detection and diagnosis using an evolving fuzzy classifier[J] . Information Sciences, 2013, 220: 64-85. 